\title{
BUILDING A CAMERA TO SEE THE FIRST GALAXIES
}

\author{
AS ASTRONOMERS LOOK DEEPER INTO SPACE, THEY REQUIRE BETTER INSTRUMENTS \\ TO DO SO. A JOINT TEAM OF SCIENTISTS AND ENGINEERS AT CARDIFF UNIVERSITY, \\ UK, AND THE NATIONAL INSTITUTE OF ASTROPHYSICS, OPTICS AND ELECTRONICS, \\ MEXICO, HAVE RISEN TO THIS CHALLENGE. WITH THE NEW TECHNOLOGY IN THEIR \\ GROUND-BREAKING CAMERA, WE COULD SOON BE LEARNING MORE ABOUT THE \\ FORMATION OF THE UNIVERSE'S BIGGEST STARS AND OLDEST GALAXIES
}

\section{TALK LIKE AN ASTRONOMER}

\section{ABSOLUTE ZERO - the lowest}

temperature possible, equal to 0 Kelvin or $-273.15^{\circ} \mathrm{C}$

\section{ELECTROMAGNETIC RADIATION}

- travelling electric and magnetic waves, commonly known as light

\section{VISIBLE SPECTRUM - the range of} wavelengths of electromagnetic radiation which can be seen by the human eye

WAVELENGTH - the distance between one wave crest to the next

Have you ever tried to imagine seeing colours that you have never seen before? Have you wished you could see with $\mathrm{x}$-ray vision or could spot things at night with infrared eyes? Astronomers do not have to imagine what it is like to see beyond the visible spectrum. They have long been finding new perspectives on stars and galaxies by tuning into different wavelengths of electromagnetic radiation. Observing the sky at a new wavelength can reveal completely new information about the Universe.
However, there are still some wavelengths that have been invisible to astronomers until recently. These wavelengths cannot be detected using standard equipment, requiring researchers to design and make their own devices if they want to capture the information that they contain. One of these 'blind spots' is in the millimetre range (around the boundary between infrared and microwaves). That is why a team of scientists and engineers, including Dr Sam Rowe at Cardiff University and Dr Victor Gómez and Marcial Tapia at the National Institute of Astrophysics, Optics and Electronics have been working on the Mexico UK Submillimetre Camera for AsTronomy, or MUSCAT.

\section{WHAT IS MUSCAT?}

MUSCAT is a camera, but you might not recognise it as one if you saw it. For a start, it looks like a metal cylinder 1 metre wide and 1 metre tall, with a window in the top and some electronics attached to the outside. Despite its size, MUSCAT does not come with its own lens, but relies on a separate piece of equipment to collect light for it. In this case, the 'lens' is a 50-metre-wide telescope called the Large Millimeter Telescope (LMT).
The job of the camera itself is to measure radiation of millimetre wavelengths collected by the LMT. To do this, a huge number of technical components are required, which have been the focus of this collaborative project. "This project is unique in that almost every aspect of the instrument has been designed, built, assembled and tested by our own engineers, researchers and students," explains Sam.

WHAT DOES THE CAMERA CONSIST OF?

Optical components filter, reflect, and guide incoming electromagnetic radiation (light) to where it needs to go. The goal of these components is to focus all the millimetre wavelengths collected by the telescope and only the millimetre wavelengths - onto the detectors. To do this, filters are used to block other wavelengths of light before an arrangement of mirrors reflects the radiation onto a region called the focal plane.

The detector is the part that measures the incoming electromagnetic radiation. MUSCAT requires a new type of very sensitive detector to measure the very faint light signals, called 
The team acknowledge RCUK and CONACYT for funding through the Newton Fund project ST/P002803/ kinetic inductance detectors (KIDs). Víctor was responsible for designing the KIDs for MUSCAT. A significant challenge for building a camera with KIDs is that they only work if they are cooled almost to absolute zero. So, a complex cooling system also had to be designed to cool the camera to 0.1 Kelvin $\left(-273.05^{\circ} \mathrm{C}\right)$.

Another challenge when it comes to measuring light at millimetre wavelengths is that all objects above absolute zero emit radiation. Because objects on Earth are much warmer than the objects in space that MUSCAT is trying to image, they emit about a million times more electromagnetic radiation at millimetre wavelengths.

This room temperature electromagnetic emission would saturate the detectors, so filters are used to keep out the excess radiation.

The final part of the camera is the readout system, which captures signals from the detector and turns them into digital data. Sam and Marcial have been developing the electronics and software to do this, allowing astronomers to analyse the new millimetre information being received from space.

\section{WHY IS THE LMT AT THE TOP OF A} MOUNTAIN IN MEXICO?

After years of designing and building, MUSCAT is now on its way to the summit of Sierra Negra to be installed at the LMT. At 4,600 metres above sea level, Sierra Negra is the fifth highest peak in Mexico. The reason for this location has everything to do with the millimetre wavelengths that the LMT was built to observe.

The millimetre part of the electromagnetic spectrum is strongly absorbed by water molecules. Therefore, humid air and clouds in the atmosphere will soak up any of these wavelengths coming from space before they reach the ground. The high altitude of the LMT means there is less atmosphere between it and space, and therefore less chance of the radiation being soaked up. Furthermore, Sierra Negra has a very dry climate, meaning the atmosphere often opens up like a 'window' into space.
WORKING TOGETHER ACROSS CONTINENTS

Scientific advancements are collaborative efforts, and the development of MUSCAT clearly highlights this. The technology transfer between institutions has allowed team members in both Cardiff and Mexico to gain new skills and knowledge while working together to build a state-of-the-art camera for astronomical observations. There were difficulties to working collaboratively with a 6-hour time difference, with researchers having to stay up late or wake early in the morning to keep in touch with their colleagues on the other side of the Atlantic. But despite this, the collaboration has been a huge success. And most importantly of all, as Marcial notes, "establishing scientific collaborations strengthens the bonds of friendship between institutions and nations."

\section{WHAT DISCOVERIES COULD MUSCAT} MAKE?

Historically, very few astronomical

observations have been from millimetre waves. However, it is estimated that about half of all the light in the Universe ever emitted by stars has been absorbed by dust and re-emitted at millimetre wavelengths. What have we been missing out on?

For a start, we will be able to see into cold clouds of interstellar dust that absorb the ultraviolet, visible and infrared wavelengths emitted by the stars. These interstellar clouds absorb then re-emit the energy back into space at millimetre wavelengths. Being able to see this radiation will help us to understand more about how very large stars form.

Furthermore, millimetre wavelengths will allow astronomers to see back in time to the earliest galaxies formed after the Big Bang. This is possible because the Universe is expanding like a balloon, meaning the light from the furthest away galaxies is effectively 'stretched', increasing its wavelength into the millimetre range.

In the years to come, hopefully MUSCAT will allow astronomers to answer many of the unanswered questions about the Universe in which we live.

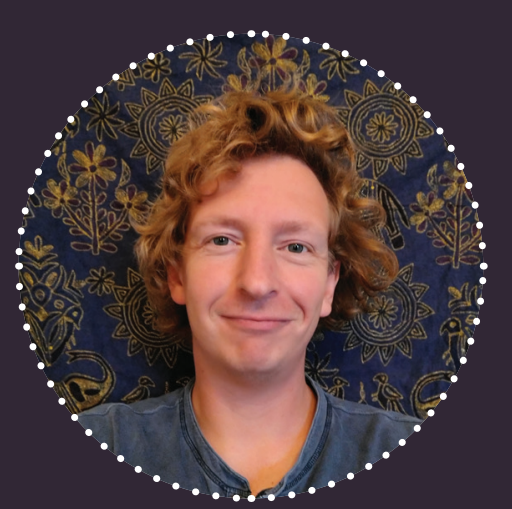

DR SAM ROWE

Cardiff University, UK

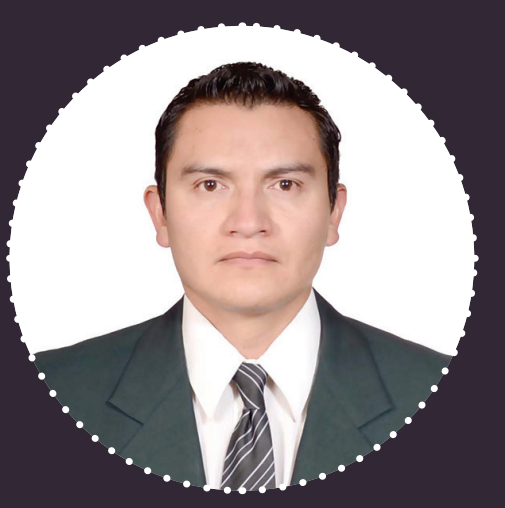

DR VÍCTOR GÓMEZ

National Institute of Astrophysics, Optics and Electronics, Mexico

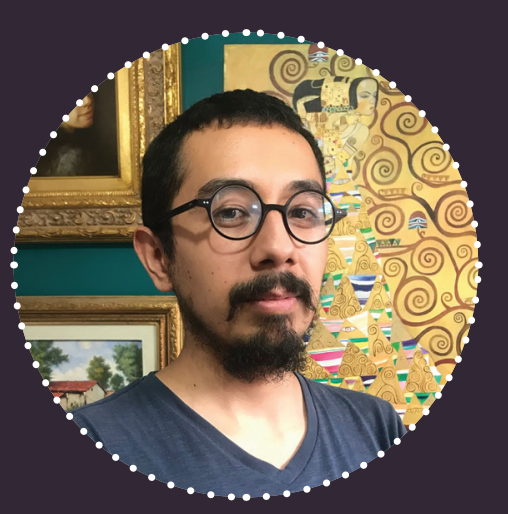

MARCIAL TAPIA

National Institute of Astrophysics, Optics and Electronics, Mexico

..........

\section{FIELD OF RESEARCH}

Astronomy Technology

\section{RESEARCH PROJECT}

Designing an astronomical camera to measure millimetre wavelength radiation

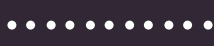

\section{FUNDERS}

Research Councils UK, Consejo Nacional de Ciencia y Tecnología, Newton Fund 


\section{ABOUT ASTRONOMY TECHNOLOGY}

Our curiosity to solve the mysteries of the night sky and find our place in the Universe has driven some of the greatest inventions in history. Today, building the tools of observational astronomy requires expertise across a wide range of fields, including physics, mechanical engineering, electronics, digital signal processing, software programming and data analysis.

The sense of wonder when looking into the sky is a strong motivation for designing the technology required to do this. "There's something very rewarding about building something that can detect light that has travelled almost halfway across the Universe from some of the most distant and ancient galaxies," says Sam. As Víctor comments, "Studying distant sources, from galaxies and star formation to planets and comets, is like taking a look into the past. This is because the light takes millions of years to reach us, so we see the galaxies as they were millions of years ago."

\section{WHY IS ASTRONOMY \\ TECHNOLOGY IMPORTANT?}

While we need observations of space to help us understand more about our place within the Universe, the technology developed for astronomy often makes its way back down to Earth to solve problems closer to home. Wireless networks now used in computers were originally developed to sharpen images from astronomical telescopes, and the $\mathrm{X}$-ray technology designed for astronomical observatories is now used to scan your luggage at the airport.

Astronomy is the oldest science, and for centuries, developments in optics and astronomy technology have led to advances in human technology. "Astronomy technology offers an idea of the scope of human ingenuity," says Marcial.

Furthermore, many of the technologies and techniques used to look into space are now being used to observe the Earth, which is more important than ever due to climate change. Satellite images using a range of different wavelengths lead to new discoveries every day about the world's oceans, forests, icecaps and atmosphere.

\section{WHAT ARE THE CHALLENGES IN} ASTRONOMY TECHNOLOGY?

The day-to-day challenges of astronomy technology involve designing and building specialist technical equipment to characterise new devices, working at ultralow temperatures with novel materials and developing computer code for automating tests and measurements. The longer-term challenges are about problem solving - it takes a lot of careful planning, organisation and experimenting to create a new instrument like MUSCAT. In the future, astronomers will aim to build more advanced telescopes with larger arrays of ultra-sensitive detectors, so there will be no lack of technical challenges for the next generation of astronomers, physicists, engineers and computer scientists.

\section{PATHWAY FROM SCHOOL TO ASTRONOMY TECHNOLOGY}

- Study STEM subjects at school such as maths, physics and computing. Languages are also useful for working on international collaborations.

- An undergraduate degree in physics or astrophysics would provide a direct route to astronomy technology, but a degree in electronics engineering or computer science could also lead there.

- Attend workshops and summer schools to broaden your knowledge of the field and find out about the opportunities available.

- Most astronomy instrumentation jobs will require a relevant master's or PhD.

MEET SAM

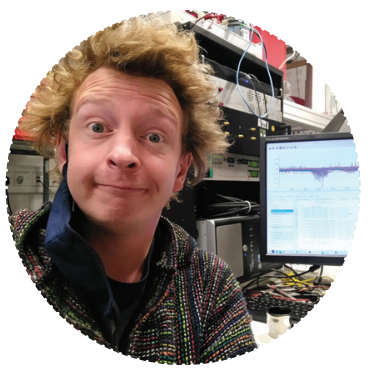

READOUT LEAD CARDIFF UNIVERSITY
Growing up, I played lots of computer games, watched lots of Star Trek and read lots of books. I loved learning new facts and particularly enjoyed flicking through countless editions of the Guinness Book of World Records. I enjoyed camping with family and friends, and still do, and I always pack a pair of binoculars for a spot of star gazing before relaxing by the campfire.

\section{There were no scientists in} my family before me, but my grandfather was a satellite television engineer, and I was always wowed by the high-tech gadgets in his workshop. And my grandmother was a big fan of alien abduction conspiracy stories which definitely engaged my critical thinking skills from a young age.

Spectacular astronomical events were all great sources of inspiration for me - the passing of Comet Hale-Bopp when I was 11, the total solar eclipse when I was 14 and the transit of Venus when I was 18.

I've worked on lots of projects during my career but completing the build of the MUSCAT instrument with such a fantastic team of collaborators is definitely my proudest achievement. Finishing my undergraduate degree and $\mathrm{PhD}$ were also proud moments for me, as was seeing Marcial successfully defending his master's thesis on which I was his co-supervisor.

I look forward to helping the team get the instrument installed and running at the telescope so we can start observations and data analysis and hopefully generate some really interesting scientific outputs. Beyond that, my ambition remains to develop more advanced technology and to use it to uncover more facts about our Universe. 
MEET VÍCTOR

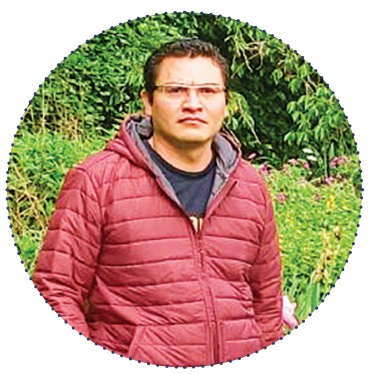

DETECTOR DESIGN LEAD NATIONAL INSTITUTE OF ASTROPHYSICS, OPTICS AND ELECTRONICS
As a child, I always liked creating things, especially electronics.

I loved knowing you could create great instruments with small devices, as practically all technology depends on electronics.

I have always liked to look at the sky and see the stars and know that they exist beyond our solar system. After finishing my degree in electronics engineering, that was the motivation to do a master's and $\mathrm{ahD}$ in astronomical instrumentation. I focused on the design of detectors, since with these small devices we can create images to observe and study the Universe.

\section{Achieving my $\mathrm{PhD}$ and} collaborating in this great project have been highlights of my career so far. I have obtained knowledge of detector design and the skills to characterize detectors. These detectors are part of the focal plane array of MUSCAT and I am eagerly waiting for the commissioning at the LMT site to make scientific observations.
One of my ambitions is to be able to design, manufacture and characterize this type of detector at the National Institute of Astrophysics, Optics and Electronics (INAOE) and seek applications both in science and in some other areas.

\section{MEET MARCIAL}

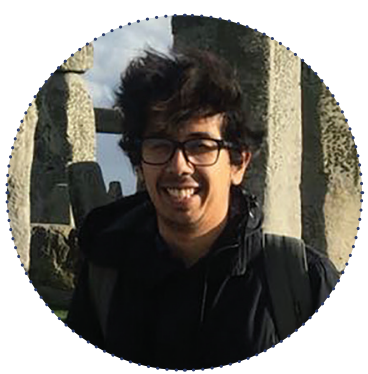

ANALYSIS PIPELINE DEVELOPER AND READOUT ENGINEER

NATIONAL INSTITUTE OF ASTROPHYSICS, OPTICS AND ELECTRONICS
I acquired a taste for astronomy from a very young age. I read with great enthusiasm an old book that my father bought in a street market which dealt with mysteries and curiosities of the world and space. There, I read about the dazzling intensity of a quasar capable of blinding the light of the galaxy that hosts it, or about black holes, objects so massive where not even light can escape its gravitational field. Reading it captivated me and instilled in me a taste for astronomy.

Thanks to the influence of my father, I acquired a taste for designing and creating machines in his carpentry workshop, which later led me to study mechatronics engineering.
A decisive event occurred during university when I participated in a scientific summer project at INAOE that allowed me to apply my engineering knowledge in astronomy. The experience of combining two of my greatest passions led me to follow the path of astronomical instrumentation.

\section{Several people influenced me} to pursue a scientific career, including my high school physics teacher, Professor Luis Gil, whose way of understanding and transmitting science is inspiring, and my graduate thesis advisor, Dr Abraham Luna, who showed me the possibility of applying engineering to astronomy.
I feel very proud to have obtained a scholarship to study for six months at the Catholic University of Chile, where I studied

astronomical instrumentation and met fabulous researchers who reinforced my conviction to follow this path.

At the moment, my ambitions are focused on successfully completing my doctorate, writing a good thesis and participating in various publications. Then, with all the experience acquired and the team formed in Mexico thanks to MUSCAT, I would like to propose, design and build the next millimetre camera for the LMT.

\section{THE MUSCAT TEAM'S TOP TIPS}

01 There are many aspects to astronomy instrumentation with lots of interesting areas in which to specialise. But it is important to start by developing a strong understanding of basic physical principles.

02 Work hard and never be afraid to ask questions. Remember, there is no such thing as a stupid question - you'll learn much more than if you keep quiet.

03 If you want to study and develop an instrument, try it no matter how difficult it is. It is very rewarding in the end when you succeed!

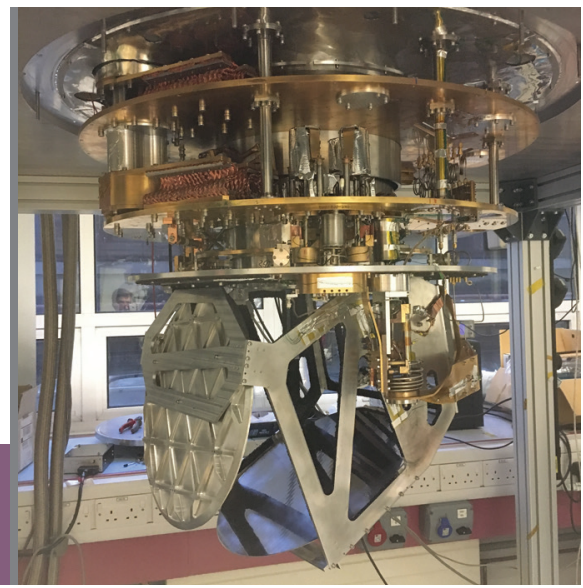

